\title{
Comparing the Hybrid Fluorescent-Radioactive Tracer Indocyanine Green-99m Tc-Nanocolloid with 99mTc-Nanocolloid for Sentinel Node Identification: A Validation Study Using Lymphoscintigraphy and SPECT/CT
}

Oscar R. Brouwer ${ }^{1}$, Tessa Buckle ${ }^{1,2}$, Lenka Vermeeren ${ }^{1}$, W. Martin C. Klop ${ }^{3}$, Alfons J.M. Balm ${ }^{3}$, Henk G. van der Poel ${ }^{4}$, Bas W. van Rhijn ${ }^{4}$, Simon Horenblas ${ }^{4}$, Omgo E. Nieweg ${ }^{5}$, Fijs W.B. van Leeuwen ${ }^{1,2}$, and Renato A. Valdés Olmos ${ }^{1}$

${ }^{1}$ Department of Nuclear Medicine, The Netherlands Cancer Institute-Antoni van Leeuwenhoek Hospital, Amsterdam, The Netherlands; ${ }^{2}$ Department of Radiology, Leiden University Medical Center, Leiden, The Netherlands; ${ }^{3}$ Department of Head and Neck Oncology and Surgery, The Netherlands Cancer Institute-Antoni van Leeuwenhoek Hospital, Amsterdam, The Netherlands; ${ }^{4}$ Department of Urology, The Netherlands Cancer Institute-Antoni van Leeuwenhoek Hospital, Amsterdam, The Netherlands; and ${ }^{5}$ Department of Surgery, The Netherlands Cancer Institute-Antoni van Leeuwenhoek Hospital, Amsterdam, The Netherlands

The purpose of this study was to compare the lymphoscintigraphic drainage patterns of a hybrid sentinel node tracer consisting of the fluorescent dye indocyanine green (ICG) and $99 \mathrm{mTc}-$ nanocolloid with the drainage pattern of 99mTc-nanocolloid alone, the current standard tracer in many European countries. Methods: Twenty-five patients with a melanoma in the head and neck region $(n=10)$, a melanoma on the trunk ( $n=$ $6)$, or penile carcinoma $(n=9)$ who were scheduled for sentinel node biopsy were prospectively included. First, the standard $99 \mathrm{~m} T \mathrm{c}-$ nanocolloid procedure was performed. After injection at the lesion site, lymphoscintigraphy was performed with a 10min dynamic study and static planar images at $10 \mathrm{~min}$ and $2 \mathrm{~h}$ after injection, followed by SPECT/CT. The same scintigraphic procedure was repeated after injection of hybrid ICG-99mTcnanocolloid the same afternoon in 10 patients or the next morning in 15 patients. The paired images of both injections were evaluated, and count rates in the sentinel nodes were calculated and compared. Sentinel nodes were surgically localized using blue dye, a $\gamma$-ray detection probe, a portable $\gamma$-camera, and a fluorescence camera. Results: Lymphatic drainage was visualized in all 25 patients using ${ }^{99 m}$ Tc-nanocolloid, leading to the identification of 66 sentinel nodes in total. These same sentinel nodes were also identified during the second scintigraphic procedure with ICG- ${ }^{99 m}$ Tc-nanocolloid. Moreover, a high correlation between the radioactive counting rates in the sentinel nodes of both scintigraphic studies was observed (mean $R^{2}=$ 0.83). Intraoperatively (4-23 $\mathrm{h}$ after the second injection), all preoperatively identified sentinel nodes could be localized using radio- and fluorescence guidance combined. In total, $95 \%$ of the sentinel nodes could be intraoperatively visualized by means of fluorescence imaging, whereas merely $54 \%$ stained blue. Ex vivo, all radioactive sentinel nodes were fluorescent and vice

\footnotetext{
Received Jan. 13, 2012; revision accepted Mar. 5, 2012.

For correspondence or reprints contact: Renato A. Valdés Olmos, The Netherlands Cancer Institute-Antoni van Leeuwenhoek Hospital, Plesmanlaan 121, Amsterdam $1066 \mathrm{CX}$, The Netherlands.

E-mail: r.valdes@nki.nl

Published online May 29, 2012.

COPYRIGHT @ 2012 by the Society of Nuclear Medicine, Inc.
}

versa. No adverse reactions were observed. Conclusion: The lymphatic drainage pattern of ICG-99mTc-nanocolloid is identical to that of $99 \mathrm{~m}$ Tc-nanocolloid. This observation, together with the added value of intraoperative fluorescence guidance, warrants wider evaluation of hybrid ICG-99mTc-nanocolloid as a tracer for sentinel node procedures.

Key Words: sentinel node; fluorescence; multimodal; image guided surgery; radiocolloid

J Nucl Med 2012; 53:1034-1040

DOI: 10.2967/jnumed.112.103127

B ased on the hypothesis of sequential tumor spread, sentinel node biopsy is increasingly being used as a staging procedure for various malignancies (1-7). In the preoperative setting, the lymphatic drainage pattern can gradually be visualized using sequential lymphoscintigraphy, enabling identification of the first tumor-draining (sentinel) lymph nodes (8). SPECT combined with CT complements lymphoscintigraphy with 3-dimensional anatomic data and can sometimes reveal additional sentinel nodes (9-11).

Intraoperative sentinel node identification traditionally relies on the combination of acoustic signals generated by a $\gamma$-ray detection probe and optical sentinel node visualization using a visible blue dye $(12,13)$. Because the probe has a limited spatial resolution and sentinel nodes do not always stain blue, sentinel nodes can be difficult to localize in areas with a complex anatomy and when sentinel nodes are near the injection site $(14,15)$. Although the incorporation of a portable $\gamma$-camera in the intraoperative procedure partially addresses these limitations by increasing the detection sensitivity and providing an intraoperative overview image of the radioactive sentinel nodes, this device does not depict the surrounding anatomic structures in the surgical field $(16-18)$. 
Near-infrared fluorescence imaging has the potential to address the drawbacks of radioguided sentinel node detection by providing better spatial resolution and allowing for real-time optical detection of the sentinel node within the surrounding anatomy (19-22). Yet, because the signal penetration of fluorescent probes is limited by tissue attenuation, radioguidance to the general area of interest is still indispensable (23). To combine the beneficial properties of both modalities, a hybrid tracer comprising the fluorescent dye indocyanine green (ICG) and the human serum albumin-based radiocolloid ${ }^{99 \mathrm{~m}} \mathrm{Tc}$-nanocolloid was developed $(24,25)$. Recently, hybrid ICG-99m Tc-nanocolloid was introduced for laparoscopic and open sentinel node biopsies in patients with prostate cancer or head and neck melanoma $(23,26,27)$.

Before this hybrid tracer can be used routinely, it is imperative to ensure that the addition of the fluorescent moieties does not alter the biologic properties of the parental radiocolloid. Therefore, the main purpose of the present study was to assess the concordance between the lymphatic drainage pattern of ${ }^{99 \mathrm{~m}} \mathrm{Tc}$-nanocolloid (the current standard in many European countries) and hybrid ICG- ${ }^{99 \mathrm{~m} T c-n a n o-}$ colloid, using lymphoscintigraphy and SPECT/CT. In addition, this study further evaluated the value of combined radio- and fluorescence-guided sentinel node biopsy in various malignancies with superficial lymphatic drainage to areas such as the groin, axilla, and neck.

\section{MATERIALS AND METHODS}

\section{Patients}

Twenty-five patients with a melanoma in the head and neck region $(n=10)$, a melanoma on the trunk $(n=6)$, or penile carcinoma $(n=9)$ who were scheduled for sentinel node biopsy were prospectively enrolled in the study. Patient characteristics are listed in Table 1. The mean age of the patients was 54 y (range, 26-74 y). All patients were clinically node-negative at the time of sentinel node biopsy. The study protocol was approved by the medical ethical committee of our institution, and all patients provided written informed consent.

\section{Tracer Preparation}

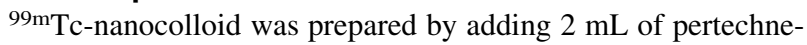
tate in saline $(\sim 1,400 \mathrm{MBq})$ to a commercial vial of nanocolloid containing $0.5 \mathrm{mg}$ of albumin colloid (GE Healthcare). After $30 \mathrm{~min}$ of incubation at room temperature, the ${ }^{99 \mathrm{~m}} \mathrm{Tc}$-nanocolloid

\section{TABLE 1}

Patient Characteristics and Lymphoscintigraphy Results

\begin{tabular}{|c|c|c|c|c|c|c|c|c|c|c|c|}
\hline \multirow[b]{2}{*}{ Patient } & \multirow[b]{2}{*}{ Age $(y)$} & \multirow[b]{2}{*}{ Sex } & \multirow[b]{2}{*}{ Primary tumor } & \multirow[b]{2}{*}{$\begin{array}{l}\text { Interval between } \\
\text { injections }(\mathrm{h})\end{array}$} & \multicolumn{3}{|c|}{ Study 1} & \multicolumn{3}{|c|}{ Study 2} & \multirow[b]{2}{*}{$R^{2}$} \\
\hline & & & & & $\begin{array}{l}\text { Dose } \\
\text { (MBq) }\end{array}$ & $\begin{array}{l}\text { SNs } \\
(n)\end{array}$ & $\begin{array}{c}\text { Basins } \\
(n)\end{array}$ & $\begin{array}{l}\text { Dose } \\
\text { (MBq) }\end{array}$ & $\begin{array}{l}\text { SNs } \\
(n)\end{array}$ & $\begin{array}{c}\text { Basins } \\
\text { (n) }\end{array}$ & \\
\hline 1 & 33 & $M$ & Melanoma trunk & 24 & 71 & 2 & 1 & 80 & 2 & 1 & * \\
\hline 2 & 66 & $\mathrm{M}$ & Melanoma trunk & 21 & 88 & 3 & 2 & 77 & 3 & 2 & 0.91 \\
\hline 3 & 26 & $\mathrm{~F}$ & Melanoma trunk & 24 & 74 & 2 & 2 & 79 & 2 & 1 & * \\
\hline 4 & 65 & $\mathrm{M}$ & Melanoma trunk & 20 & 78 & 2 & 1 & 80 & 2 & 2 & * \\
\hline 5 & 50 & $\mathrm{~F}$ & Melanoma trunk & 5 & 71 & 2 & 2 & 68 & 2 & 2 & * \\
\hline 6 & 30 & $\mathrm{~F}$ & Melanoma trunk & 4 & 68 & 3 & 3 & 73 & 3 & 3 & 0.99 \\
\hline 7 & 74 & $\mathrm{M}$ & Head/neck melanoma & 23 & 61 & 3 & 3 & 70 & 3 & 3 & 0.99 \\
\hline 8 & 55 & $\mathrm{M}$ & Head/neck melanoma & 3 & 59 & 2 & 2 & 76 & 2 & 2 & * \\
\hline 9 & 32 & $\mathrm{~F}$ & Head/neck melanoma & 2.5 & 54 & 1 & 1 & 67 & 1 & 1 & * \\
\hline 10 & 39 & $\mathrm{M}$ & Head/neck melanoma & 4.5 & 87 & 2 & 2 & 86 & 2 & 2 & * \\
\hline 11 & 70 & $\mathrm{~F}$ & Head/neck melanoma & 22 & 64 & 3 & 3 & 67 & 3 & 3 & 0.93 \\
\hline 12 & 32 & $\mathrm{~F}$ & Head/neck melanoma & 2.5 & 64 & 3 & 2 & 72 & 3 & 2 & 0.85 \\
\hline 13 & 49 & $M$ & Head/neck melanoma & 23 & 67 & 1 & 1 & 60 & 1 & 1 & * \\
\hline 14 & 66 & $\mathrm{~F}$ & Head/neck melanoma & 20 & 61 & 2 & 2 & 58 & 2 & 2 & * \\
\hline 15 & 56 & $\mathrm{~F}$ & Head/neck melanoma & 2.5 & 82 & 6 & 6 & 98 & 6 & 6 & 0.58 \\
\hline 16 & 67 & $\mathrm{~F}$ & Head/neck melanoma & 22 & 74 & 2 & 2 & 96 & 2 & 2 & * \\
\hline 17 & 57 & $\mathrm{M}$ & Penile carcinoma & 2.5 & 80 & 2 & 2 & 87 & 2 & 2 & * \\
\hline 18 & 61 & $\mathrm{M}$ & Penile carcinoma & 2.5 & 69 & 5 & 2 & 57 & 5 & 2 & 0.96 \\
\hline 19 & 72 & $\mathrm{M}$ & Penile carcinoma & 19 & 70 & 3 & 2 & 73 & 3 & 2 & 0.93 \\
\hline 20 & 72 & $\mathrm{M}$ & Penile carcinoma & 24 & 61 & 3 & 2 & 83 & 3 & 2 & 0.71 \\
\hline 21 & 48 & $M$ & Penile carcinoma & 20 & 70 & 3 & 1 & 59 & 3 & 1 & 0.51 \\
\hline 22 & 34 & $\mathrm{M}$ & Penile carcinoma & 20 & 69 & 3 & 2 & 71 & 3 & 2 & 0.99 \\
\hline 23 & 64 & $\mathrm{M}$ & Penile carcinoma & 23 & 67 & 3 & 1 & 66 & 3 & 1 & 0.99 \\
\hline 24 & 71 & $\mathrm{M}$ & Penile carcinoma & 20 & 75 & 3 & 2 & 81 & 3 & 2 & 0.49 \\
\hline 25 & 65 & $\mathrm{M}$ & Penile carcinoma & 2.5 & 83 & 2 & 2 & 74 & 2 & 2 & * \\
\hline
\end{tabular}

${ }^{*}$ Correlation between counting rates measured $2 \mathrm{~h}$ after each injection (could be calculated only in patients with $>2 \mathrm{SNs}$ ).

Study 1 = first examination, using ${ }^{99 m T c-n a n o c o l l o i d ; ~ s t u d y ~} 2$ = second examination, using ICG_99mTc-nanocolloid; SN = sentinel node; $R^{2}=$ correlation of sentinel node counting rates in both studies. 
solution ( $\mathrm{pH} \mathrm{6-7)} \mathrm{was} \mathrm{exposed} \mathrm{to} \mathrm{air} \mathrm{via} \mathrm{a} \mathrm{needle} \mathrm{to} \mathrm{remove} \mathrm{any}$ excess reactive elements. Subsequently, a $0.25-\mathrm{mg}$ dose of ICG (ICG-Pulsion; Pulsion Medical Systems) was added to form hybrid ICG- ${ }^{99 m}$ Tc-nanocolloid as described previously (23). All preparations were performed using good manufacturing practices and with the approval of The Netherlands Cancer Institute's pharmacist.

\section{Preoperative Procedure and Image Analysis}

To determine the concordance between the lymphatic drainage patterns of ICG- ${ }^{99 \mathrm{~m}} \mathrm{Tc}$-nanocolloid and ${ }^{99 \mathrm{~m}} \mathrm{Tc}-$ nanocolloid, both tracers were injected in consecutive order in the same patients, and the lymphoscintigraphic findings of both tracers were directly compared. First, ${ }^{99 \mathrm{~m}}$ Tc-nanocolloid was injected, in a volume of $0.4 \mathrm{~mL}$ containing $0.05 \mathrm{mg}$ of human serum albumin. Before tracer injection, the planned sites of injection were carefully marked with an indelible felt-tip pen (Fig. 1). In the melanoma patients, 4 injections were placed intradermally around the scar of the primary melanoma excision. For penile carcinoma, the same dosage was intradermally administered divided into 3 injections proximally around the tumor. The mean radioactivity dose of the first injection calculated on the basis of net administered doses was $71 \mathrm{MBq}$ (range, 54-88 MBq). Immediately after injection, anterior and lateral dynamic images were obtained with a dualhead $\gamma$-camera (Symbia T; Siemens) over $10 \mathrm{~min}$ to visualize the lymphatic flow and to identify lymph nodes on a direct lymphatic drainage pathway. Subsequently, static planar images were acquired at $15 \mathrm{~min}$. Two hours after injection of ${ }^{99 \mathrm{~m}} \mathrm{Tc}$-nanocolloid, delayed planar images were obtained to further differentiate firstechelon nodes from higher-echelon nodes and to identify sentinel nodes in other basins. In the same session, SPECT/CT (Symbia T; Siemens) was performed. The lymph nodes draining directly from the injection site were classified as sentinel nodes $(8)$. When there were multiple visible nodes without visible afferent vessels, the first node appearing in the basin was considered to be the sentinel node.

The same afternoon (10 patients: $1-\mathrm{d}$ protocol) or the next morning (15 patients: $2-d$ protocol), the complete scintigraphic sequence was repeated after injection of hybrid ICG- ${ }^{99 \mathrm{~m}} \mathrm{Tc}-$ nanocolloid. Shortly before the injection, a 5-min static image was obtained as a point of reference for the second injection in 6 patients. Hybrid tracer administration was then performed at the locations previously marked on the skin, by the same nuclear physician, using a similar tracer concentration $(0.05 \mathrm{mg}$ of human serum albumin in $0.4 \mathrm{~mL}$; mean, $74 \mathrm{MBq}$; range, 57-98 MBq). The mean interval between the 2 injections was $19 \mathrm{~h}$ (median, $21 \mathrm{~h}$; range, 2.5-24 h, Table 1). Paired images of both injections were evaluated with regard to the similarity of the depicted draining lymph node basins and the location and number of the sentinel nodes. Counting rates (maximum counts per pixel) were measured from the planar anterior images at $2 \mathrm{~h}$ after each injection and the reference images before the second injection using regions of interest drawn around the sentinel nodes. Trendline-based linear regression correlations (Excel; Microsoft) were used to establish the correlation between the radioactive counting rates of both scintigraphic studies in patients with more than 2 preoperatively identified sentinel nodes.

\section{Intraoperative Procedure}

Shortly before surgery, $1.0 \mathrm{~mL}$ of patent blue dye (Laboratoire Guerbet) was injected in all patients except those with melanoma located on the face to prevent nonesthetic long-lasting skin marks by the blue dye. Next, a portable $\gamma$-camera (Sentinella; Oncovision) was used to guide the skin incisions and to obtain a preexcision overview image of the sentinel nodes. Intraoperatively, the sentinel nodes were first pursued using the acoustic guidance provided by a $\gamma$-ray detection probe (Neoprobe; Johnson \& Johnson Medical). During this initial exploration, attempts were made to visually detect the sentinel nodes via the blue dye (by eye) and the hybrid tracer's fluorescent component (ICG) using a handheld near-infrared fluorescence camera (PDE; Hamamatsu). After excision of each sentinel node, a postexcision image was acquired with the portable $\gamma$-camera to ensure its complete removal.

\section{Ex Vivo Analyses and Histopathologic Examination}

Excised lymph nodes were postoperatively analyzed for the presence of a radioactive or fluorescent signal using the portable $\gamma$-camera and an ex vivo fluorescence camera system (IVIS 200; Caliper Lifesciences), respectively. All harvested nodes were fixed in formalin, bisected, embedded in paraffin, and cut at a minimum of 6 levels at 50- to $150-\mu \mathrm{m}$ intervals. Pathologic evaluation included hematoxylin-eosin and immunohistochemical staining (Anticytokeratin; CAM 5.2; Becton Dickinson).
FIGURE 1. Comparison of lymphatic drainage pattern of ${ }^{99 m T c-n a n o c o l l o i d ~ a n d ~}$ ICG-99mTC-nanocolloid in a patient with melanoma on right posterior flank. (A) First ${ }^{99 m T c-}$ nanocolloid was injected after planned sites of injection were carefully marked with indelible felt-tip pen. (B) Planar lymphoscintigram $2 \mathrm{~h}$ after injection shows 4 deposits at injection site (i) and 2 sentinel nodes (SN), 1 in each axilla. (C) Axial SPECT/CT image reveals anatomic information for both sentinel nodes. (D) Same patient was injected with ICG-99mTc-nanocolloid $24 \mathrm{~h}$ later at previously marked locations. (E) Planar lymphoscintigram $2 \mathrm{~h}$ after injection reveals same 2 axillary sentinel nodes. (F) Axial SPECT/CT image confirms that both sentinel nodes are at exactly the same locations as in first scintigraphic study using ${ }^{99 m T c-n a n o c o l l o i d . ~}$

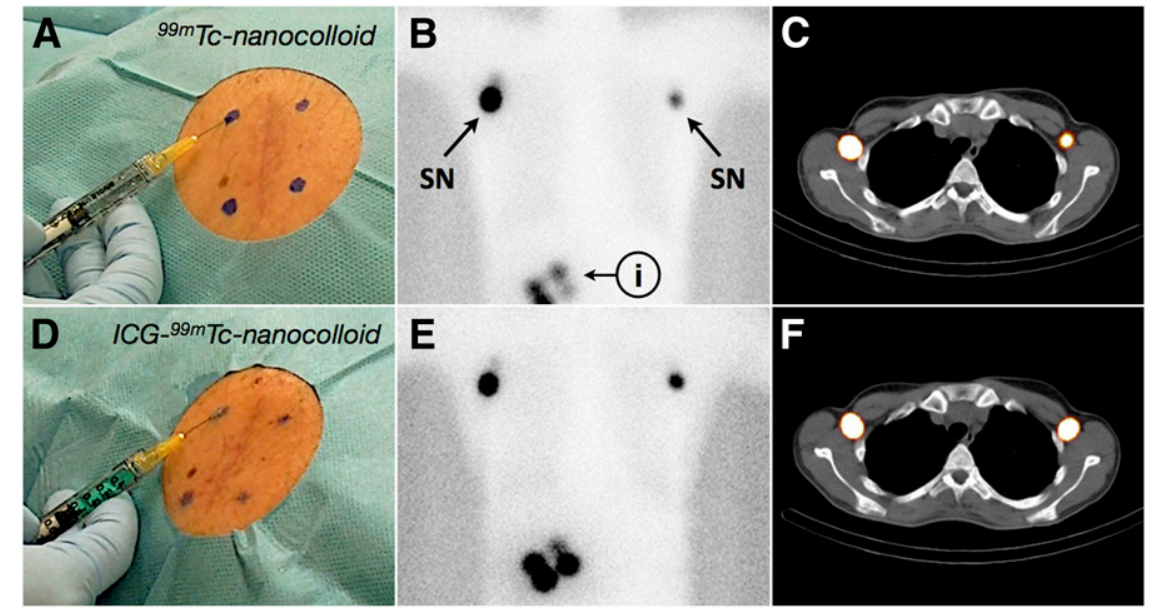




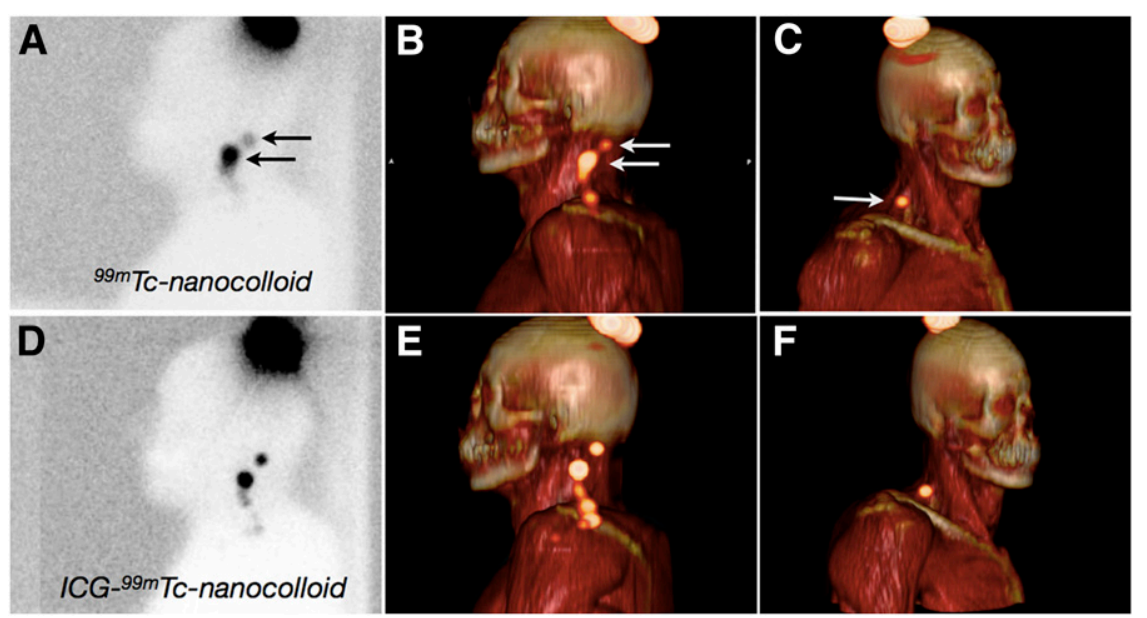

FIGURE 2. Comparison of Iymphatic drainage pattern of ${ }^{99 \mathrm{mTC}} \mathrm{Tc}$-nanocolloid and ICG-99mTc-nanocolloid in a patient with a melanoma on parietal scalp. (A) Planar lymphoscintigram $2 \mathrm{~h}$ after injection of ${ }^{99 \mathrm{mTC}} \mathrm{Tc}$ nanocolloid shows 2 sentinel nodes (arrows) in left neck region, with second-echelon activity in caudal direction. (B) Threedimensional volume-rendered SPECT/CT image reveals 2 sentinel nodes (arrows) in level $\mathrm{V}$ of left side of neck and secondechelon node located more caudally. (C) Three-dimensional volume-rendered SPECT/ CT image reveals sentinel node in level $\mathrm{V}$ on right side (which was also visible on anterior planar image, not shown). (D) Planar lymphoscintigram $2 \mathrm{~h}$ after injection of ICG-99nTCnanocolloid (23 $\mathrm{h}$ after ${ }^{99 n} \mathrm{Tc}$-nanocolloid injection) shows same 2 sentinel nodes on left side, with increased higher-echelon activity. (E) Three-dimensional volume-rendered SPECT/CT image shows sentinel nodes in same location as in first scintigraphic study, with more notable higher-echelon activity. (F) Injection of ICG-99nTcnanocolloid also led to identification of same sentinel node on right side, as seen on 3-dimensional volume-rendered SPECT/CT image.

\section{RESULTS}

\section{Preoperative Image Analysis}

Lymphoscintigraphy and SPECT/CT after injection of 99m Tc-nanocolloid showed at least 1 sentinel node in all 25 patients. The conventional lymphoscintigrams depicted 65 sentinel nodes, of which $89 \%$ were visualized on the early static planar images. SPECT/CT showed the anatomic location of these nodes and revealed 1 additional sentinel node that was not visualized on the conventional lymphoscintigrams. These 66 sentinel nodes were distributed over 51 nodal basins (Table 1; average of 2.6 sentinel nodes per patient). In the 10 patients with a melanoma in the head and neck region, 25 sentinel nodes were observed in various neck lymph node basins $(n=17)$, the parotid gland $(n=$ $2)$, the submandibular region $(n=2)$, and the suboccipital region $(n=3)$. The 6 patients with a melanoma on the trunk had 14 sentinel nodes in the axillae $(n=9)$ and groin $(n=2)$. In the 9 patients with penile carcinoma, 27 sentinel nodes were visualized in the groin $(n=16)$.

The second scintigraphic study using ICG- ${ }^{99 \mathrm{~m} T c-n a n o-}$ colloid yielded an identical drainage pattern, with the same number of sentinel nodes in the corresponding nodal basins for all patients (Table 1; Figs. 1-3). A comparison of the radioactive counting rates in the individual sentinel nodes at $2 \mathrm{~h}$ after both injections revealed a strong correlation $\left(R^{2}\right)$ of $0.85 \pm 0.20$ for the patients injected twice on the same day (1-d protocol) and $0.83 \pm 0.19$ for the patients within the 2-d protocol (Table 1). In the 1-d protocol, radioactive counting rates measured $2 \mathrm{~h}$ after the second injection (ICG- ${ }^{99 \mathrm{~m}} \mathrm{Tc}$-nanocolloid) were higher than counting rates measured $2 \mathrm{~h}$ after the first injection ( ${ }^{99 \mathrm{~m}} \mathrm{Tc}$-nanocolloid) in $90 \%$ of the sentinel nodes. For the 2-d protocol, in which more of the original activity has decayed at the time of the second injection, this was 66\%. Furthermore, in the 6 patients for whom a reference image had been acquired shortly before the second scintigraphic procedure (both 1and 2-d protocols), an increased radioactive counting rate was found in each sentinel node $2 \mathrm{~h}$ after the second injection (average, 85\%; range, 47\%-96\%). This indicates that in both the 1-d and the 2-d protocol, ICG- ${ }^{99 \mathrm{~m}} \mathrm{Tc}-$-nanocolloid drained to the very sentinel nodes identified during the first scintigraphic study (using ${ }^{99 \mathrm{~m} T c-n a n o c o l l o i d) . ~}$

\section{Intraoperative Results}

On average, sentinel node biopsy started $6 \mathrm{~h}$ after injection of the hybrid tracer (range, 4-23 h; median, $5 \mathrm{~h}$,

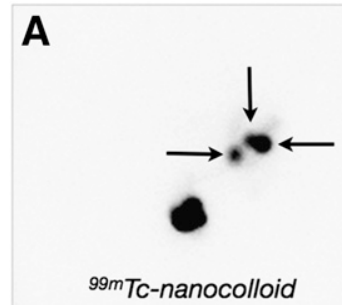

B

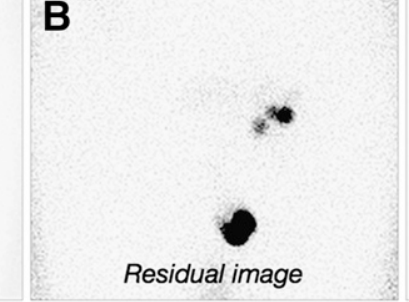

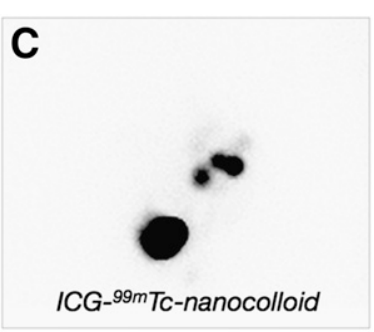

FIGURE 3. Comparison of lymphatic drainage pattern of ${ }^{99 m T c-n a n o c o l l o i d ~ a n d ~}$ ICG_99mTC-nanocolloid in a patient with a penile carcinoma. (A) Planar lymphoscintigram $2 \mathrm{~h}$ after injection of ${ }^{99 \mathrm{~m} T \mathrm{~T}-\text {-nanocolloid }}$ shows drainage to 3 sentinel nodes in left inguinal region (arrows). (B) Next morning, residual image before ICG-99mTc-nanocolloid injection shows decreased activity in 3 nodes due to radioactive decay. (C) Planar lymphoscintigram $2 \mathrm{~h}$ after injection of ICG-99mTc-nanocolloid shows drainage to same 3 sentinel nodes in left inguinal region (later confirmed by SPECT/CT). 


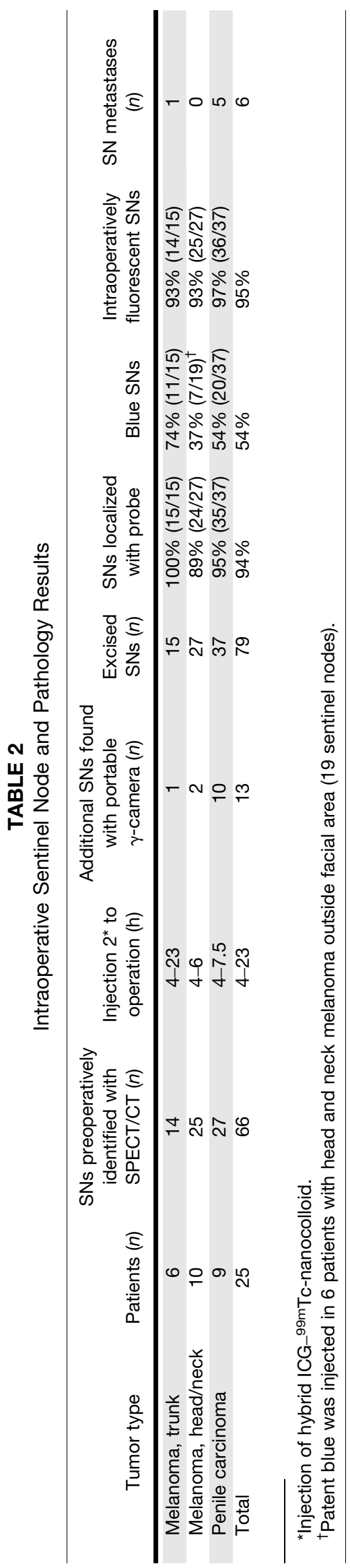

Table 2). Image acquisition using the portable $\gamma$-camera ranged between $20 \mathrm{~s}$ and $1 \mathrm{~min}$. Near-infrared fluorescence imaging using the handheld fluorescence camera was performed in real time. Intraoperatively, all scintigraphically visualized sentinel nodes could be localized and excised using radio- and fluorescence guidance combined. In 6 patients, postexcision imaging with the portable $\gamma$-camera identified considerable residual radioactivity at the original location of the sentinel node, resulting in the pursuit and removal of 13 additional sentinel nodes. Of the total of 79 sentinel nodes, 74 (94\%) could be localized using the $\gamma$-ray detection probe. The remaining 5 nodes were identified using the fluorescence camera (Table 2). Three of these sentinel nodes concerned melanomas in the temporal region and were in front of the ear. Although these sentinel nodes could be distinguished using the portable $\gamma$-camera, the overwhelming radioactive signal from the nearby injection site hampered probe guidance. Two sentinel nodes in the inguinal region in penile carcinoma patients were difficult to localize using the probe and portable $\gamma$-camera because of the low radioactive counting rate in the sentinel nodes compared with the high background.

A total of 75 sentinel nodes ( $95 \%$ ) could be visualized by fluorescence imaging after initial surgical exploration (exemplified in Fig. 4), whereas only 54\% of the sentinel nodes were stained when patent blue was used (21 patients, Table 2). The 4 sentinel nodes that eluded fluorescent imaging were probably not sufficiently exposed to enable detection of the fluorescent signal. Nevertheless, a fluorescent signal could be visualized in all radioactive sentinel nodes (including the above 4) ex vivo using the more sensitive IVIS fluorescence imaging system. In addition, none of the excised nodes exclusively contained fluorescence. This finding underlines the stability of the ICG- ${ }^{99 \mathrm{~m}} \mathrm{Tc}$-nanocolloid complex.

Histopathologic examination revealed metastases in 6 excised sentinel nodes in 4 patients (Table 2). The use of ICG- ${ }^{99 \mathrm{~m}}$ Tc-nanocolloid was not associated with adverse reactions.

\section{DISCUSSION}

This study showed that the hybrid radioactive and fluorescent tracer ICG- ${ }^{99 \mathrm{~m}} \mathrm{Tc}$-nanocolloid has the same lymphatic drainage pattern as ${ }^{99 \mathrm{~m}} \mathrm{Tc}$-nanocolloid, the current standard radiopharmaceutical in most European countries. Lymphoscintigraphy and SPECT/CT after injection of ICG- ${ }^{99 m}$ Tc-nanocolloid did not reveal any sentinel nodes at other locations, and all preoperatively identified sentinel nodes were found to contain ICG after excision. This confirms that ICG- ${ }^{99 \mathrm{~m}} \mathrm{Tc}$-nanocolloid drains to the same sentinel nodes as ${ }^{99} \mathrm{~m}$ Tc-nanocolloid and accumulates in the sentinel nodes accordingly. The high correlation between counting rates measured in the sentinel nodes on the lymphoscintigrams $2 \mathrm{~h}$ after each tracer injection in both the 1-d and the 2-d protocol further substantiates these findings. Combined with the absence of adverse reactions, these findings validate the use of ICG- ${ }^{99}{ }^{9} \mathrm{Tc}-$ nanocolloid 


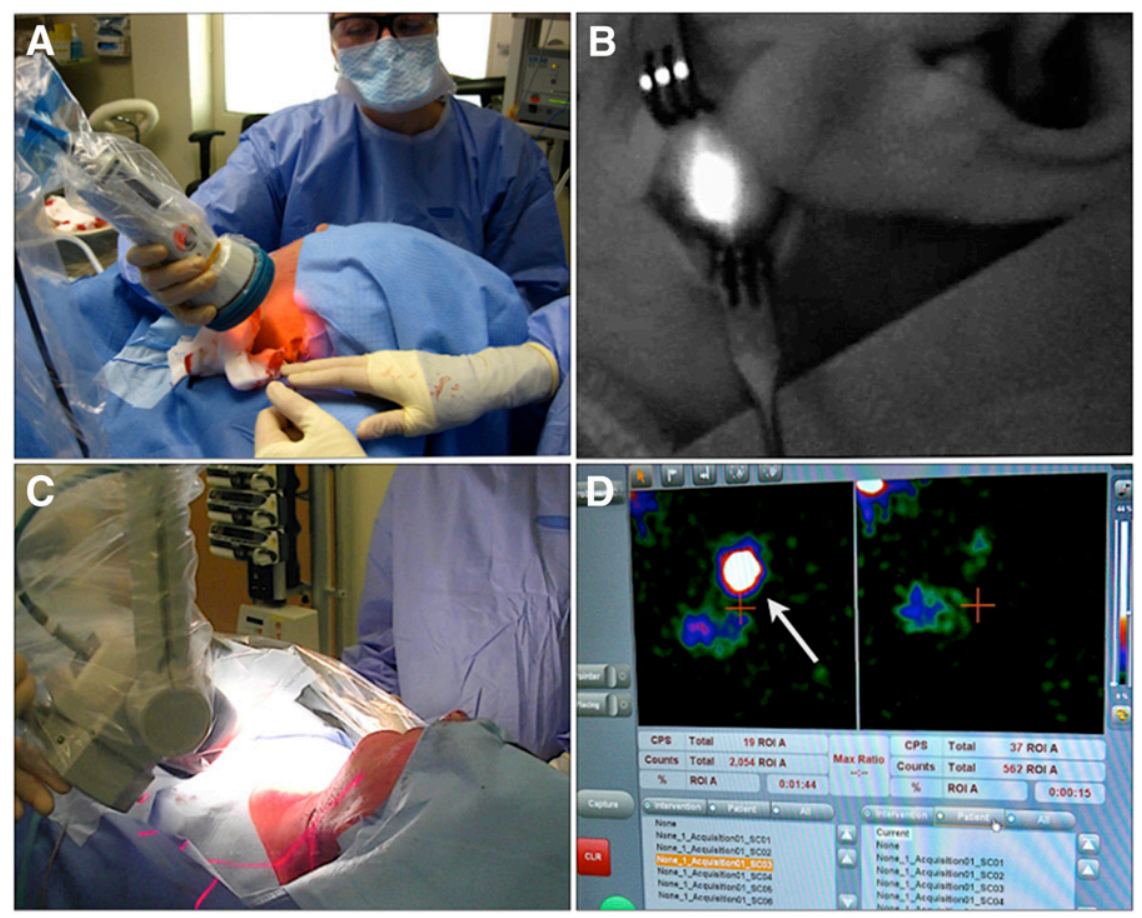

FIGURE 4. Combined intraoperative radio- and fluorescence-guided sentinel node biopsy in patient with head and neck melanoma. (A) After initial exploration guided by $\gamma$-probe and portable $\gamma$-camera, handheld fluorescence camera is used to visualize sentinel node. (B) Near-infrared fluorescence image shows exact location and margins of sentinel node within exposure of infraauricular sentinel node. (C) Portable $\gamma$-camera is used to make pre- and postexcision image of radioactive sentinel nodes. (D) By comparing preexcision (left) and postexcision (right) image on screen of portable $\gamma$-camera, surgeon can confirm successful removal of each sentinel node (arrow) in operating room. as a tracer for preoperative lymphatic mapping and sentinel node identification.

The reproducibility of lymphoscintigraphy with ${ }^{99 \mathrm{~m}} \mathrm{Tc}-$ nanocolloid when performed twice has been studied by us in the past for penile carcinoma and melanoma $(28,29)$. Although our present results are in agreement with the $100 \%$ reproducibility rate found for penile carcinoma, a discordance in lymphoscintigraphy results after the second scintigraphic study could have been anticipated for melanoma, as Kapteijn et al. found a reproducibility rate of $88 \%$ for lymphoscintigraphy repeated after 2-4 wk in melanoma patients (29). A possible explanation for the high reproducibility observed in the current study might lie in the significantly shorter interval between the 2 injections. The placement of the second injection at the exact locations previously marked on the skin is also likely to have had a positive influence on the high reproducibility rate found in the current study (Fig. 1).

In recent years, the fluorescent dye ICG has been evaluated as a single agent for intraoperative lymphatic mapping and sentinel node identification $(19,20,30)$. Like vital blue dyes, ICG is a small-particle organic dye and migrates quickly through the lymphatic system, resulting in a relatively short detection window after injection and the necessity for careful timing to intraoperatively identify the sentinel nodes. In practice, this may require the use of a 50-fold higher ICG dosages than the one used in the current study (20). Moreover, rather than visualizing lymph flow with fluorescence imaging, the hybrid approach individually illuminates the very nodes identified on lymphoscintigrams and SPECT/CT. The high rate of fluorescent sentinel nodes visualized in the operating room (95\%), compared with the relatively low percentage of sentinel nodes that were stained blue (54\%), underlines how the fluorescent extension provided by $\mathrm{ICG}-{ }^{99 \mathrm{~m}} \mathrm{Tc}$-nanocolloid can improve intraoperative visualization of the sentinel nodes. On top of this, the time window for fluorescent detection of the sentinel nodes was extended up to $23 \mathrm{~h}$ after injection in the current study. The improved tissue penetration that fluorescence imaging offers over vital blue dyes and the high resolution that can be obtained compared with $\gamma$-tracing modalities help enhance optical identification of the sentinel nodes. This advantage proved to be especially helpful when high radioactive background signals impeded sentinel node localization using the probe, in accordance with previous findings in patients with head and neck melanoma or prostate cancer $(23,27)$.

In the present study, the application of hybrid ICG${ }^{99 \mathrm{~m} T c}$-nanocolloid was successfully extended to anatomic areas such as the axilla and the groin. This success encourages further extension of this technique to other areas where radioguided surgery can be challenging. The introduction of hybrid tracers also poses new technologic challenges for manufacturers of imaging systems. In the current study, separate devices for radioguided and optical sentinel detection were used. In the future, the development of hybrid devices combining the 2 techniques may further improve the logistics in daily clinical practice.

\section{CONCLUSION}

The lymphatic drainage pattern of hybrid ICG- ${ }^{99 \mathrm{~m}} \mathrm{Tc}-$ nanocolloid is identical to that of ${ }^{99} \mathrm{~m} \mathrm{Tc}$-nanocolloid, and 
no adverse reactions were observed. These findings, together with the added value of intraoperative fluorescence guidance, warrant further evaluation of hybrid ICG- ${ }^{99 \mathrm{~m}} \mathrm{Tc}-$ nanocolloid as a tracer for sentinel node procedures.

\section{DISCLOSURE STATEMENT}

The costs of publication of this article were defrayed in part by the payment of page charges. Therefore, and solely to indicate this fact, this article is hereby marked "advertisement" in accordance with 18 USC section 1734.

\section{ACKNOWLEDGMENTS}

This research was supported, in part, by a KWF translational research award (grant PGF 2009-4344) and a NWO VIDI grant (STW BGT 11271). We thank the melanoma surgeons, head and neck surgeons, urologists, operation department practitioners, and technicians of the nuclear medicine department for their contribution. We also gratefully acknowledge Professor Theo J.M. Ruers for kindly providing the fluorescence camera system. No other potential conflict of interest relevant to this article was reported.

\section{REFERENCES}

1. Jansen L, Koops HS, Nieweg OE, et al. Sentinel node biopsy for melanoma in the head and neck region. Head Neck. 2000;22:27-33.

2. Saha S, Dan AG, Bilchik AJ, et al. Historical review of lymphatic mapping in gastrointestinal malignancies. Ann Surg Oncol. 2004;11:245S-246S.

3. Aikou T, Kitagawa Y, Kitajima M, et al. Sentinel lymph node mapping with GI cancer. Cancer Metastasis Rev. 2006;25:269-277.

4. Meinhardt W, Valdés-Olmos RA, van der Poel HG, Bex A, Horenblas S. Laparoscopic sentinel node dissection for prostate carcinoma: technical and anatomical observations. BJU Int. 2008;102:714-717.

5. Leijte JAP, Hughes B, Graafland NM, et al. Two-center evaluation of dynamic sentinel node biopsy for squamous cell carcinoma of the penis. J Clin Oncol. 2009;27:3325-3329.

6. Bex A, Vermeeren L, de Windt G, Prevoo W, Horenblas S, Valdés Olmos RA. Feasibility of sentinel node detection in renal cell carcinoma: a pilot study. Eur J Nucl Med Mol Imaging. 2010;37:1117-1123.

7. Brouwer OR, Valdés Olmos RA, Vermeeren L, Hoefnagel CA, Nieweg OE, Horenblas S. SPECT/CT and a portable gamma-camera for image-guided laparoscopic sentinel node biopsy in testicular cancer. J Nucl Med. 2011;52: 551-554.

8. Nieweg OE, Tanis PJ, Kroon BB. The definition of a sentinel node. Ann Surg Oncol. 2001;8:538-541.

9. van der Ploeg IMC, Valdés-Olmos RA, Kroon BBR, Nieweg OE. The hybrid SPECT/CT as an additional lymphatic mapping tool in patients with breast cancer. World J Surg. 2008;32:1930-1934.

10. Vermeeren L, van der Ploeg IMC, Valdés Olmos RA, et al. SPECT/CT for preoperative sentinel node localization. J Surg Oncol. 2010;101:184-190.

11. Vermeeren L, Valdés Olmos RA, Klop WMC, et al. SPECT/CT for sentinel lymph node mapping in head and neck melanoma. Head Neck. 2011;33:1-6.
12. Nieweg OE, Jansen L, Kroon BB. Technique of lymphatic mapping and sentinel node biopsy for melanoma. Eur J Surg Oncol. 1998;24:520-524.

13. Kapteijn BA, Nieweg OE, Liem I, et al. Localizing the sentinel node in cutaneous melanoma: gamma probe detection versus blue dye. Ann Surg Oncol. 1997;4:156160.

14. Tanis PJ, Nieweg OE, van den Brekel MWM, Balm AJM. Dilemma of clinically node-negative head and neck melanoma: outcome of "watch and wait" policy, elective lymph node dissection, and sentinel node biopsy-a systematic review. Head Neck. 2008;30:380-389.

15. Kroon BK, Horenblas S, Meinhardt W, et al. Dynamic sentinel node biopsy in penile carcinoma: evaluation of 10 years experience. Eur Urol. 2005;47:601-606.

16. Vermeeren L, Valdés-Olmos RA, Meinhardt W, Horenblas S. Intraoperative imaging for sentinel node identification in prostate carcinoma: its use in combination with other techniques. J Nucl Med. 2011;52:741-744.

17. Vermeeren L, Valdés-Olmos RA, Klop WMC, Balm AJM, van den Brekel MWM. A portable gamma-camera for intraoperative detection of sentinel nodes in the head and neck region. J Nucl Med. 2010;51:700-703.

18. Vermeeren L, Valdés Olmos RA, Meinhardt W, et al. Intraoperative radioguidance with a portable gamma camera: a novel technique for laparoscopic sentinel node localisation in urological malignancies. Eur J Nucl Med Mol Imaging. 2009;36: 1029-1036.

19. Mieog JSD, Troyan SL, Hutteman M, et al. Toward optimization of imaging system and lymphatic tracer for near-infrared fluorescent sentinel lymph node mapping in breast cancer. Ann Surg Oncol. 2011;18:2483-2491.

20. Hutteman M, Mieog JSD, van der Vorst JR, et al. Randomized, double-blind comparison of indocyanine green with or without albumin premixing for nearinfrared fluorescence imaging of sentinel lymph nodes in breast cancer patients. Breast Cancer Res Treat. 2011;127:163-170.

21. Crane LMA, Themelis G, Arts HJG, et al. Intraoperative near-infrared fluorescence imaging for sentinel lymph node detection in vulvar cancer: first clinical results. Gynecol Oncol. 2011;120:291-295.

22. Hayashi T, Furukawa H, Oyama A, et al. Sentinel lymph node biopsy using realtime fluorescence navigation with indocyanine green in cutaneous head and neck/lip mucosa melanomas. Head Neck. 2012;34:758-761.

23. van der Poel HG, Buckle T, Brouwer OR, Valdés Olmos RA, van Leeuwen FWB. Intraoperative laparoscopic fluorescence guidance to the sentinel lymph node in prostate cancer patients: clinical proof of concept of an integrated functional imaging approach using a multimodal tracer. Eur Urol. 2011;60:826-833.

24. van Leeuwen AC, Buckle T, Bendle G, et al. Tracer-cocktail injections for combined pre- and intraoperative multimodal imaging of lymph nodes in a spontaneous mouse prostate tumor model. J Biomed Opt. 2011;16:016004.

25. Buckle T, van Leeuwen AC, Chin PTK, et al. A self-assembled multimodal complex for combined pre- and intraoperative imaging of the sentinel lymph node. Nanotechnology. 2010;21:355101.

26. Buckle T, Brouwer OR, Valdés Olmos RA, van der Poel HG, van Leeuwen FWB. Relationship between intraprostatic tracer deposits and sentinel lymph node mapping in prostate cancer patients. J Nucl Med. 2012;53:1026-1033.

27. Brouwer OR, Klop WM, Buckle T, et al. Feasibility of sentinel node biopsy in head and neck melanoma using a hybrid radioactive and fluorescent tracer. Ann Surg Oncol. December 30, 2011 [Epub ahead of print].

28. Kroon BK, Valdés-Olmos RA, van Tinteren H, Nieweg OE, Horenblas S. Reproducibility of lymphoscintigraphy for lymphatic mapping in patients with penile carcinoma. J Urol. 2005;174:2214-2217.

29. Kapteijn BA, Nieweg OE, Valdés Olmos RA, et al. Reproducibility of lymphoscintigraphy for lymphatic mapping in cutaneous melanoma. J Nucl Med. 1996;37:972-975.

30. Bredell MG. Sentinel lymph node mapping by indocyanin green fluorescence imaging in oropharyngeal cancer: preliminary experience. Head Neck Oncol. 2010;2:31-37. 